\title{
A discrete element approach for modelling the compression of crop stems
}

\author{
Tom Leblicq ${ }^{\mathrm{a}, *}$, Bart Smeets ${ }^{\mathrm{a}}$, Herman Ramon ${ }^{\mathrm{a}}$, Wouter Saeys ${ }^{\mathrm{a}}$ \\ ${ }^{a} K U$ Leuven - University of Leuven, Department of Biosystems (BIOSYST), Division of \\ Mechatronics, Biostatistics and Sensors (MeBioS), Kasteelpark Arenberg 30, 3001 \\ Leuven, Belgium
}

\begin{abstract}
The discrete element method (DEM) offers a powerful tool for simulating the interactions of large numbers of particles. Recently, DEM was used to simulate the interactions of tubular particles. While the existing linear elastic and Hertzian contact models can approximate the reversible compression for small deformations, they are inadequate for larger deformations. Here, the force-deformation behaviour is highly non-linear and plastic. In this study, data based contact models were developed for crop stems. These models combine realistic deformation behaviour with a minimal number of model parameters. Furthermore, the effect of plastic deformation and damage was incorporated in the model. The contact models were successfully used to validate, through comparison of simulations and measurements, individual stem and bulk compression. A good agreement was found between both. These validated DEM contact models for compression of crop stems allow to simulate the processing of large numbers of crop stems.
\end{abstract}

Keywords: DEM, Simulation, Compression, Contact model, Crop stem,

\footnotetext{
${ }^{*}$ Corresponding author.

Tel.: +32 16377 116; E-mail address: tom.leblicq@biw.kuleuven.be
} 


\section{Biomass}

\section{Introduction}

The importance of simulations for the optimization of various processes

3 is increasing because simulations can provide a huge potential in terms of 4 process understanding and development of machines. Development costs can 5 be reduced by evaluating machine adjustments in early stages via computer 6 simulations before building costly and complex prototypes. This simulation 7 approach also enables the investigation of new and unconventional ideas with 8 acceptable effort (Jünemann et al., 2013). To obtain realistic simulations, 9 both the machine and the material to be processed should be simulated with sufficient accuracy. In the case of biomaterial processing, the biological variation adds an extra challenge.

\subsection{Discrete element modelling of crop stems}

When modelling the behaviour of a collection of particles, Discrete Element Modeling (DEM) is a logical choice as it allows to describe the behaviour of each particle through its interactions with the other particles and the system elements (Tijskens et al., 2003). In this way, the influence of 
particle properties (both geometrical and mechanical properties) and boundary conditions (shape and motion of machine parts) can be assessed with a set of in silico experiments that can be run in parallel (Lenaerts et al., 2014). To obtain reliable simulation results, the virtual stems must have realistic geometries and deform realistically during contact. The stems should, therefore be compressible in both longitudinal and radial direction and also bendable in every direction. However, few reports have been published on particles with large aspect-ratio, as simulating the movement and collisions of these particles is considerably more complex than for rigid spherical particles.

In the last years, tubular particles were created by a number of researchers (Ross \& Klingenberg, 1997; Favier et al., 1999; Grof et al., 2007; Kattenstroth et al., 2011; Geng et al., 2011; Guo et al., 2012b, 2013a,b; Nguyen et al., 2013; Grof \& Štěpánek, 2013; Jünemann et al., 2013; Kajtar \& Loebe, 2014; Nan et al., 2014; Lenaerts et al., 2014; Leblicq et al., 2014, 2015a). The accuracy of the simulations with these particles varies greatly. Ross \& Klingenberg (1997) studied the dynamics of flowing suspensions of rigid and flexible fibres composed of linked rigid bodies. They demonstrated that the method can reproduce known dynamical behaviour of both types of fibres in a flow field. Favier et al. (1999) developed a method for representing smooth-surfaced, axi-symmetrical particles using overlapping spheres of arbitrary size. Virtual straw stems were developed by Kattenstroth et al. (2011) by connecting spheres. The stems in this research were, however, rigid and unbendable. The same method was used to create rigid fibres and rodlike particles (Grof et al., 2007; Grof \& Štěpánek, 2013; Nan et al., 2014). Flexible filamen- 
tous particles composed of chains of rigid bodies connected through ball and socket joints were developed by Geng et al. (2011); Guo et al. (2013a,b); Grof \& Štěpánek (2013). Jünemann et al. (2013) and Kajtar \& Loebe (2014) created bendable crop stems by connecting spheres with cylindrical bonds. Guo et al. (2012b) compared glued-spheres particles and true cylindrical particles and concluded that for smooth particles the most realistic results are obtained with cylindrical particles. Guo et al. (2012a) also described methods for more optimal contact detection and dense packing of cylindrical particles. Lenaerts et al. (2014) created segmented bendable straw stems by connecting rigid cylinders with flexible configurable bonds. They used the DEMeter++ software (Tijskens et al., 2003) to simulate grain-straw separation and validated the results with measurements. Leblicq et al. (2014, 2015a) improved the bending model of Lenaerts et al. (2014) and obtained tubular particles (crop stems as well as metal and plastic tubes) with realistic bending and buckling behaviour.

\subsection{Contact models for tubular particles in DEM}

The discrete element method uses the Newton-Euler equations of motion to compute the translatory and rotational movements of every particle in the simulation. Due to these movements, particles make contact and interact. Interacting particles are allowed to virtually overlap. The normal forces which particles exert on each other are determined by a contact model (based on this virtual overlap and potentially on the contact history). Contact models for calculating normal and tangential forces are usually analogues models based on spring, damper and friction elements (Jünemann et al., 2013). 
Contact forces are typically decomposed into a normal and tangential component, with respect to the contact surface. The normal component of the contact force acting on a particle is generally described as a function of the virtual overlap $(\delta)$ and its time derivative $(\dot{\delta})$ (Tijskens et al., 2003). The simplest model assumes a linear elastic component and a linear viscous damping.

$$
F_{n}(\delta, \dot{\delta})=k_{1} \delta+\gamma_{1} \dot{\delta}
$$

For viscoelastic materials, a non-linear model, based on Hertz contact law can be derived (Tijskens et al., 2003):

$$
F_{n}(\delta, \dot{\delta})=k_{2} \delta^{3 / 2}+\gamma_{2} \delta^{1 / 2} \dot{\delta}
$$

Tangential contact forces arise when two particles take part in an oblique collision or when contacting particles are rotating relative to each other. The simplest contact model in the tangential direction describes Coulomb friction with a viscous damper enforcing the no-slip regime. As for impact in the normal direction, realistically behaving contact models in the tangential direction also require elastic and viscous terms (Tijskens et al., 2003).

These basic contact models were also used to model the interactions between tubular particles. Grof et al. (2007), Nguyen et al. (2013), Jünemann et al. (2013), Kajtar \& Loebe (2014) and Leblicq et al. (2015a) used linear spring-damper systems. Guo et al. (2012b), Nan et al. (2014) and Lenaerts et al. (2014) used Hertz contact models. All studies used Coulomb friction. 


\subsection{Limitations of the existing contact models}

Although it has been shown that stem deformation processes are definitely non-linear (Leblicq et al., 2015b), in DEM the processes have typically been assumed to be linear. Only for the first, linear elastic part of the forcedeformation curve this assumption is acceptable. At larger deformations, ovalisation, buckling and cracking of the particles takes place. This results in a highly non-linear and plastic deformation behaviour. Plastic deformation implies that stems will respond differently to re-compression or further deformation, which is ignored by the DEM contact models. The bulk behaviour of a collection of stems is the sum of the behaviours of the individual stems. Incorrect stem behaviour could thus result in incorrect simulation results and incorrect decisions for machine optimisation. Therefore there is a need for DEM contact models that can realistically describe the deformation behaviour of crop stems and that takes plastic deformation into account.

\subsection{Objectives of this study}

To the knowledge of the authors, no studies have been conducted to define DEM contact models for tubular particles describing the contact behaviour realistically both for undeformed and damaged stems. Therefore, the general objective of this study was to create and validate these contact models. In order to achieve this goal, there are some specific objectives. First, the forces required to deform crop stems need to be measured. Then, the deformation of individual stems has to be modelled using data based models. Afterwards, the interactions between individual stems and between stems and plates (representing a machine part) need to be modelled. Finally, the contact models need to be validated both on stem and bulk level. 


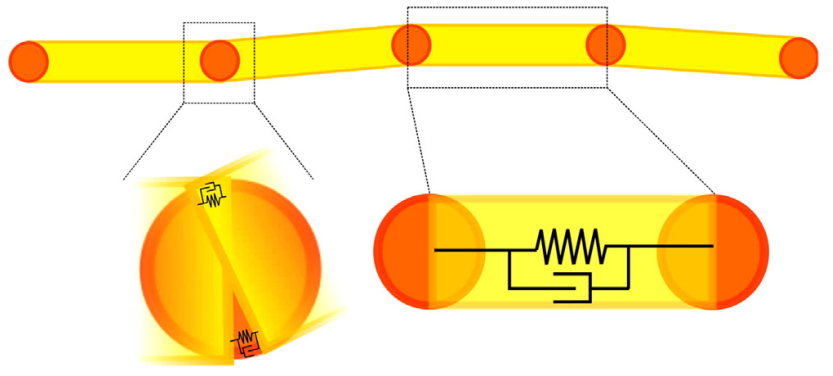

Fig. 1. Bendable straw in DEMeter++ (Lenaerts et al., 2014)

\section{Material and methods}

In this study, we started from the segmented crop stems developed by Lenaerts et al. (2014) in DEMeter ++ (Fig. 1). Each segment consists of a capsule connected to its adjacent capsules by joints. The joints are responsible for the flexibility of the particle. In each joint, a number of springdampers $\left(k_{b}, c_{b}\right)$ is placed which creates a moment that counteracts bending forces. The tensile stiffness of each segment of the straw particle is provided by one spring and damper $\left(k_{t}, c_{t}\right)$ per segment. The Werner and Haff model is used to model the tangential contact forces (friction) (Haff \& Werner, 1986). When stems interact, they virtually overlap and exert normal forces on each other. An extra spring-damper system $\left(k_{c}, c_{c}\right)$ is responsible for these forces. However, in this study the value of the contact spring is not a constant (as is the case in the linear elastic and in the Hertz model), but depends on the overlap $(\delta)$ and the previous deformations $(d)$ of the particle $\left(k_{c}=f(\delta, d)\right)$. The normal force is then:

$$
F_{n}(d, \delta, \dot{\delta})=F_{n, \text { spring }}+F_{n, \text { damper }}=k_{c} \delta-c_{c} \dot{\delta}
$$


The dampers prevent the elements from oscillating, dissipate energy and stabilize the simulation.

\subsection{Measurements of the mechanical properties of stems}

The values for the normal spring force were determined experimentally. The deformations imposed during the measurements were chosen as such that the range of deformations covers the deformations during processing. Two types of interactions occur: stem-stem interactions and stem-machine interactions. These interactions were measured with the set-ups shown in Fig. 2. Both types of measurements were conducted with a single column testing machine (Type LS1 Material Tester, LLoyd materials testing, West Sussex, UK) at a speed of $1 \mathrm{~mm} / \mathrm{s}$. In Fig. 2(a) the set-up for measuring the interaction between a stem and a plate is displayed. When the top plate moves down the stem is compressed and the required force is recorded with a 50 N loadcell (Type LS Loadcell 50N, LLoyd Instruments, West Sussex, UK). In Fig. 2(b) the set-up for measuring the compression of a stem due to the interaction with a stem-shaped object is shown. The stem is placed on a metal bar that has a diameter corresponding to that of an average wheat stem (3.5 mm, Leblicq et al. (2015b)). The plunger has the same dimensions. The stem is thus compressed between two metal stems.

To determine the effect of plastic deformation and damage, the measurements are not performed in one run, but step-wise. In every cycle the plunger (either plate or metal stem) moves down an additional $0.1 \mathrm{~mm}$ relative to the previous cycle and then returns to the initial position. The deformation is thus incrementally increased (Fig. 3 (black)). 


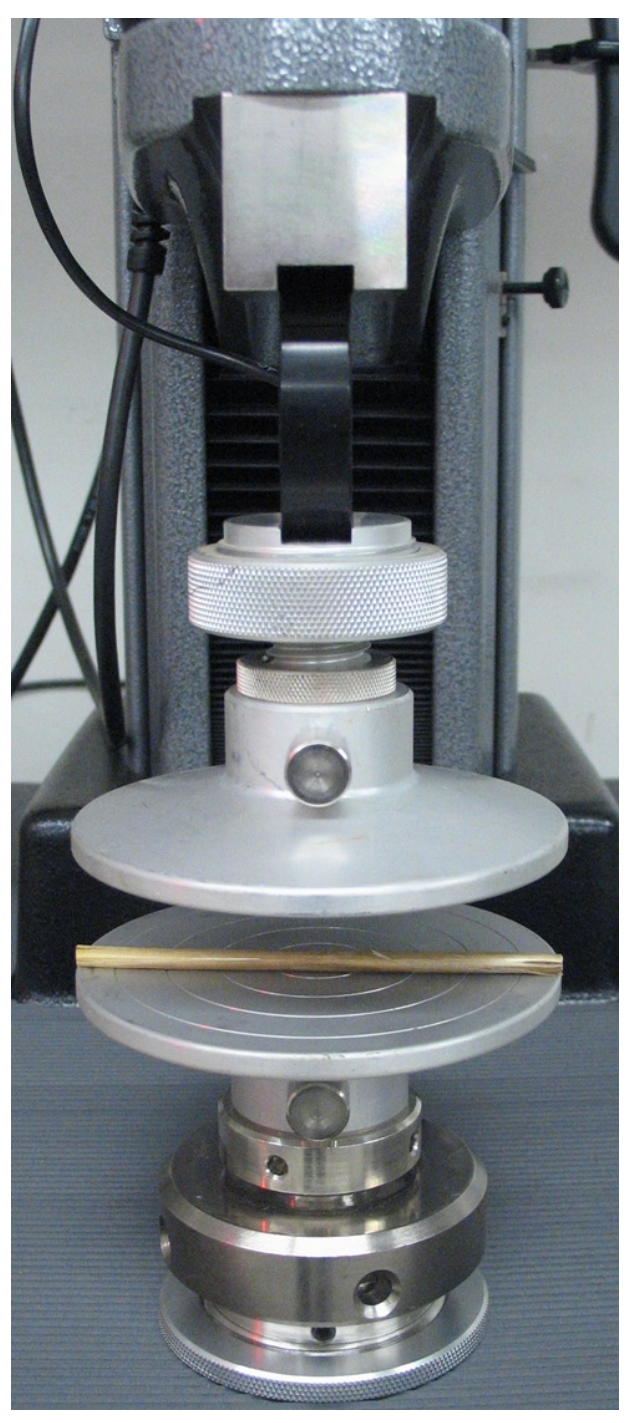

(a)

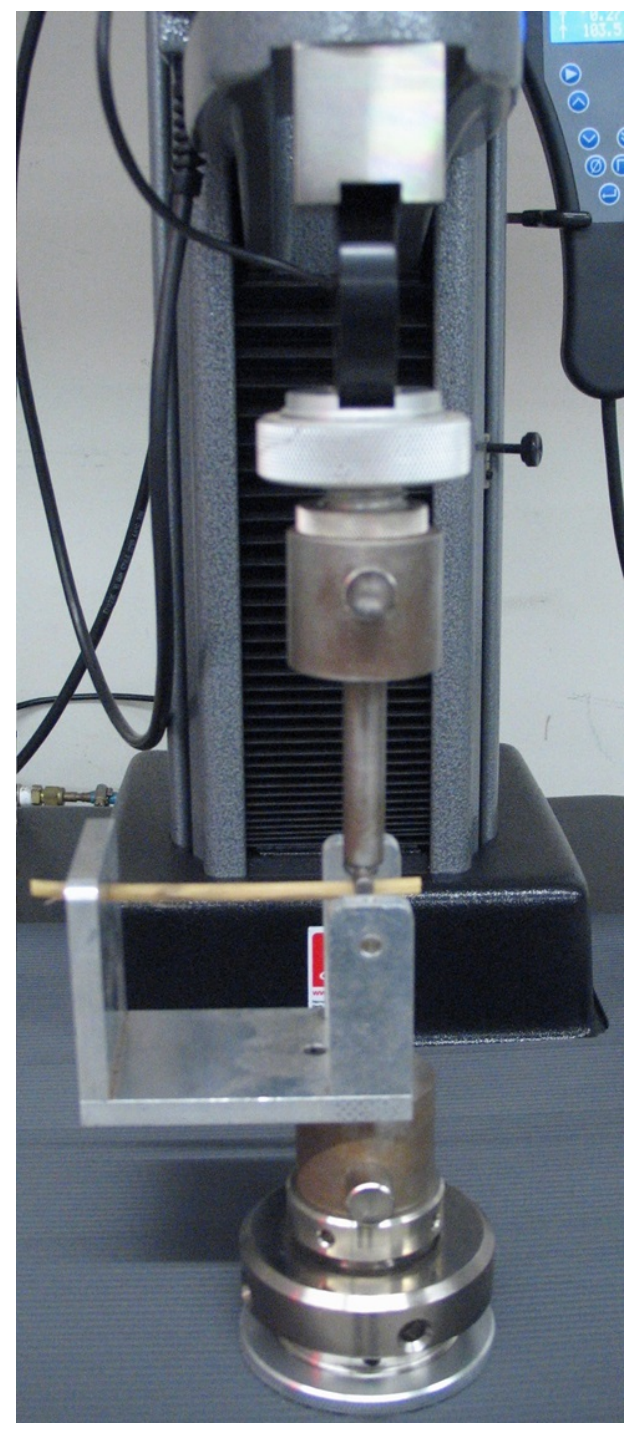

(b)

Fig. 2. Set-up for measuring stem compression

(a) stem-plate interaction, (b) stem-stem interaction 


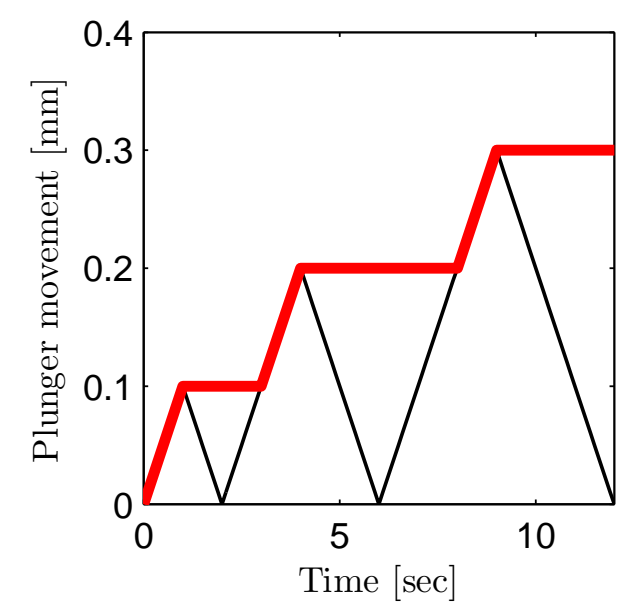

Fig. 3. black: plunger movement, red: maximum historical deformation (MHD)

To identify the variability of the deformation behaviour thirty measurements were performed on wheat stalks for each set-up. The stalks were randomly harvested by hand from one field in northern France in August 2014. Prior to the measurements the stems were cut to a length of $5 \mathrm{~cm}$. All internodes were used.

\subsection{From measured data to contact model}

The measured force-deformation data can be used to determine the contact forces $\left(F_{n, \text { spring }}\right.$ in Eq. (3)) of interacting particles. For this, the data are first processed and look up tables (LUT's) are made from the data (section 2.2.2). These LUT's are used to develop contact models. First, the interactions between stems and rigid structures are modelled. Due to the rigidity of the structure only the stems will deform. Afterwards the interaction between different stems is modelled. Here both stems will be (plastically) deformed. The extend to which each stem deforms is dependent on the relative stiffness. 


\subsubsection{Data processing}

During processing, the data points with negative indentation are removed (the plunger is not making contact with the stem). The data is divided into two groups. The data where the plunger is moving down and the stems get deformed and the data where the plunger is retracting. For each measured data point the maximum historical deformation (MHD) is determined (Fig. 3 (red)). During the first cycle, while the plunger is moving down, the MHD is increasing. When the plunger retracts, the MHD stays constant and is equal to the maximum deformation of the first cycle. During the second cycle, the MHD will only increase after the plunger has exceeded the deformation of the first cycle. The MHD is thus always equal to or larger than the indentation. Each data point consist thus of three values: indentation, force and MHD. Each pair of values for the MHD and the indentation corresponds to a value of the force. This relation is used to make LUT's from the data.

\subsubsection{Look up tables}

An equidistant grid was formed by the vectors of the MHD and the indentation. Linear interpolation was then used to determine the value of the force for each grid point. The normal force can, therefore, also be determined for intermediate values of the MHD and the indentation that were not measured. For all thirty stem-stem measurements and for all thirty stem-plate measurements, look up tables were built.

\subsubsection{Determination of deformation forces from the LUT's}

When stems in DEM interact, they overlap. This virtual overlap corresponds to the measured indentation. Each segment of the virtual stems has 
a memory, in which the maximum historical overlap/deformation (MHD) is stored. In every time step of the simulation and for each segment the overlap with neighbouring segments is calculated. From this overlap and from the stored MHD, the corresponding force can be obtained from the look up table for that stem.

\subsubsection{Switching between look up tables}

Depending on whether the deformation increases or decreases a different LUT is used (e.g. the LUT in Fig. 9(a) can be used for increasing indentation and the LUT in Fig. 9(b) for decreasing indentation). Only taking the previous time step into account to determine whether the deformation is increasing or decreasing is insufficient. High frequency vibrations would cause a constant switching between LUT's. Therefore, not only the indentation from the previous time step, but also a weighted change in indentation $(d \delta)$ is stored per contact. This weighted change of indentation is calculated as suggested by Janert (2013):

$$
d \delta_{t}=\frac{d \delta_{t-1}+\left(\frac{\delta_{t}-\delta_{t-1}}{\alpha}\right)}{1+\frac{1}{\alpha}}
$$

The weighted change of indentation $\left(d \delta_{t}\right)$ is a function of the previously calculated weighted change of indentation $\left(d \delta_{t-1}\right)$ and the difference between the overlap in the current and the previous time step $\left(\delta_{t}-\delta_{t-1}\right)$. The parameter $\alpha$ determines the weight of the current time step in the calculation of $d \delta_{t}$. A higher value of $\alpha$ makes $d \delta_{t}$ less sensitive to vibrations. When $d \delta_{t}$ is positive the indentation is increasing. When $d \delta_{t}$ is negative the indentation is decreasing. The value of $d \delta_{t}$ determines thus which LUT has to be used. 


\subsubsection{Contact mechanics}

Using LUT's, we now have virtual stems that behave realistically when compressed. This does, however, not mean that the stems interact realistically. The contact force is indeed not the sum of the compression forces of both particles separately, nor is it the average of both. Also, the deformation cannot evenly be distributed over both particles. Particles with less deformation resistance (e.g. stems with smaller wall thickness or stems which have already been deformed) will deform more and sustain more plastic deformation. This again makes it more likely that they will be damaged more during future interactions. This process will continue until a stem is completely crushed and the stem walls make contact. The resistance to deformation is then strongly increased. The extent to which each stem is deformed and damaged thus depends on the relative resistance to deformation.

The virtual overlap in DEM corresponds to physical deformation. In reality, however, stems are not evenly deformed. In this way, also the overlap can not be evenly distributed over the two virtual stems. The stem with the least resistance to deformation is attributed the largest part of the overlap $(a \delta)$, where $a$ is the percentage of the overlap for that stem. The second stem will contain the remaining part of the overlap. This part is equal to $(1-a) \delta$. The sum of the overlap of the two stems is, as a result, equal to the total overlap.

$$
a \delta+(1-a) \delta=\delta
$$

The forces between two stems (determined by the stored MHD of each stem 
and the part of the overlap that each particle receives) should however, according to Newton's third law, always be equal in magnitude (but opposite in direction). Each time particles interact, this distribution of the overlap has to be determined.

The determination of the distribution of the overlap corresponds to finding a value for $a$ such that the forces of the two stems $\left(F_{1}\right.$ and $\left.F_{2}\right)$ are equal.

$$
F_{N 1}=F_{N 2}
$$

The forces $F_{N 1}$ and $F_{N 2}$ of both stems are a function of the mechanical properties (the LUT's), the MHD's ( $M H D_{1}$ and $M H D_{2}$ ), and the virtual overlaps $(a \delta$ and $(1-a) \delta)$.

$$
F_{N 1}=L U T_{1}\left(M H D_{1}, a \delta\right) \quad F_{N 2}=L U T_{2}\left(M H D_{2},(1-a) \delta\right)
$$

The bisection method is used to solve Eq. (6) and thus to determine the value of $a, F_{N 1}$ and $F_{N 2}$. Afterwards, it is determined whether the current overlap of each particle is larger than the MHD of that particle. If this is the case, the MHD is updated.

\subsection{DEM parameters}

Apart from the contact model parameters, all parameters of the bendable stems have been defined similar to those in Lenaerts et al. (2014). The parameter values are listed in Table 1.

\subsection{Stem simulations}

To validate the contact models, stem simulations corresponding to the stem measurements from section 2.1, were conducted. The virtual stems 
Table 1. DEM parameters

\begin{tabular}{llll}
\hline Parameter & Value & Unit & Meaning \\
\hline$k_{b}$ & 0.75 & $\mathrm{~N} / \mathrm{m}$ & spring for bending \\
$c_{b}$ & $2.5 e^{-4}$ & $\mathrm{~kg} / \mathrm{s}$ & damper for bending \\
$k_{t}$ & 100 & $\mathrm{~N} / \mathrm{m}$ & spring for tension \\
$c_{t}$ & 0.1 & $\mathrm{~kg} / \mathrm{s}$ & damper for tension \\
$c_{c}$ & 0.1 & $\mathrm{~kg} / \mathrm{s}$ & damper for compression \\
$\mu$ & 0.3 & - & friction coefficient \\
$c_{\mu}$ & 10 & $\mathrm{~kg} / \mathrm{s}$ & damper for friction \\
\hline
\end{tabular}

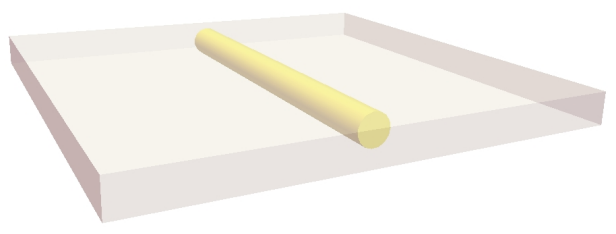

(a)

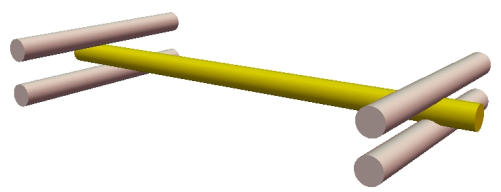

(b)

Fig. 4. Stem simulations

(a) Simulation of stem-plate interaction (equivalent to Fig. 2(a)), (b) Simulation of stem-stem interaction (equivalent to Fig. 2(b))

were given diameters equal to those from the measured stems (Table 2). The stems were compressed by a virtual metal plunger (both the plate and the metal stem, Fig. 4). The plunger was given a LUT for steel. This LUT was built from the stress-strain curve of steel (Rasmussen, 2003). The forces that are required for an equal amount of deformation are 40 times higher for steel than for wheat straw (Mohsenin, 1986). This will result in an almost complete deformation of the stem and almost no deformation of the plunger. 
The overlap is thus nearly entirely for the stem. In these simulations, the compression was also performed stepwise as was done in the experiments.

\subsection{Bulk simulations}

To validate the contact model on a bulk level, the compression of fifty stems to $50 \mathrm{~N}$ was simulated. Each virtual stem consisted of five segments and had a total length of $5 \mathrm{~cm}$. Each stem was given two pairs of LUT's. One pair for the stem-stem interaction (both increasing and decreasing indentation) and one pair for the stem-plate interaction. The LUT's were randomly chosen from the thirty LUT's that were created from the stem measurements. The stems were placed parallel to each other on the bottom of a compression box (6 $\mathrm{cm}$ wide, $6 \mathrm{~cm}$ long and $10 \mathrm{~cm}$ high). Before compression, the box was shaken two times with an amplitude of $10 \mathrm{~cm}$ and a frequency of $4 \mathrm{~s}^{-1}$ to randomise the orientation of the stems (Fig. 5(a)). This initialisation method was chosen because it could both be simulated and reproduced in validation experiments. After the randomisation, the stems were compressed at a speed of $1 \mathrm{~mm} / \mathrm{s}$ and the force on the plunger was recorded (Fig. 5(b)). This simulation cycle (random assignment of stem properties, initialisation and compression) was repeated twenty times.

\subsection{Bulk measurements}

To validate the bulk simulations, twenty bulk measurements were conducted. The same conditions were applied. The compression box had the same dimensions. The fifty stems were first placed parallel in the box and then shaken for initialisation (Fig. 6(a)). After randomisation, the stems were compressed at a rate of $1 \mathrm{~mm} / \mathrm{s}$ (Fig. 6(b)). The stems were obtained 


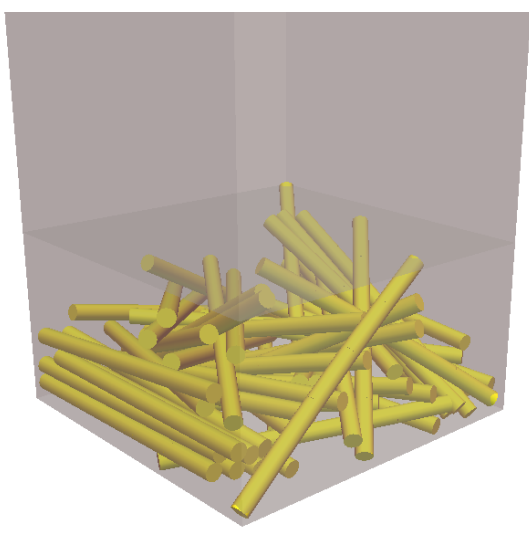

(a)

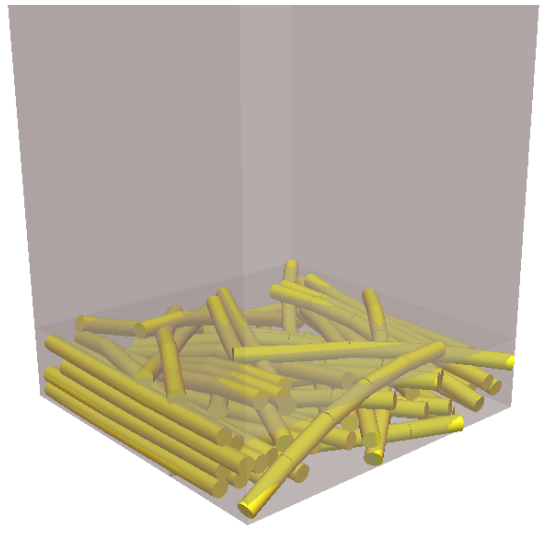

(b)

Fig. 5. Bulk simulations

(a) stem orientations after initialisation (equivalent to Fig. 6(a)), (b) bulk compression (equivalent to Fig. 6(b))

from the same field as the stems from the stem measurements. Prior to the measurements, the stems were cut to a length of $5 \mathrm{~cm}$. All internodes were used.

\subsection{Modelling bulk compression}

Due to the randomization of the stem orientations during initialization, a one on one comparison of the simulation and the measurement results was not possible. To compare the results, an existing compression model was fitted to the stress-strain data. The following exponential model was used (O’Dogherty, 1989):

$$
\sigma=m e^{n \epsilon}
$$




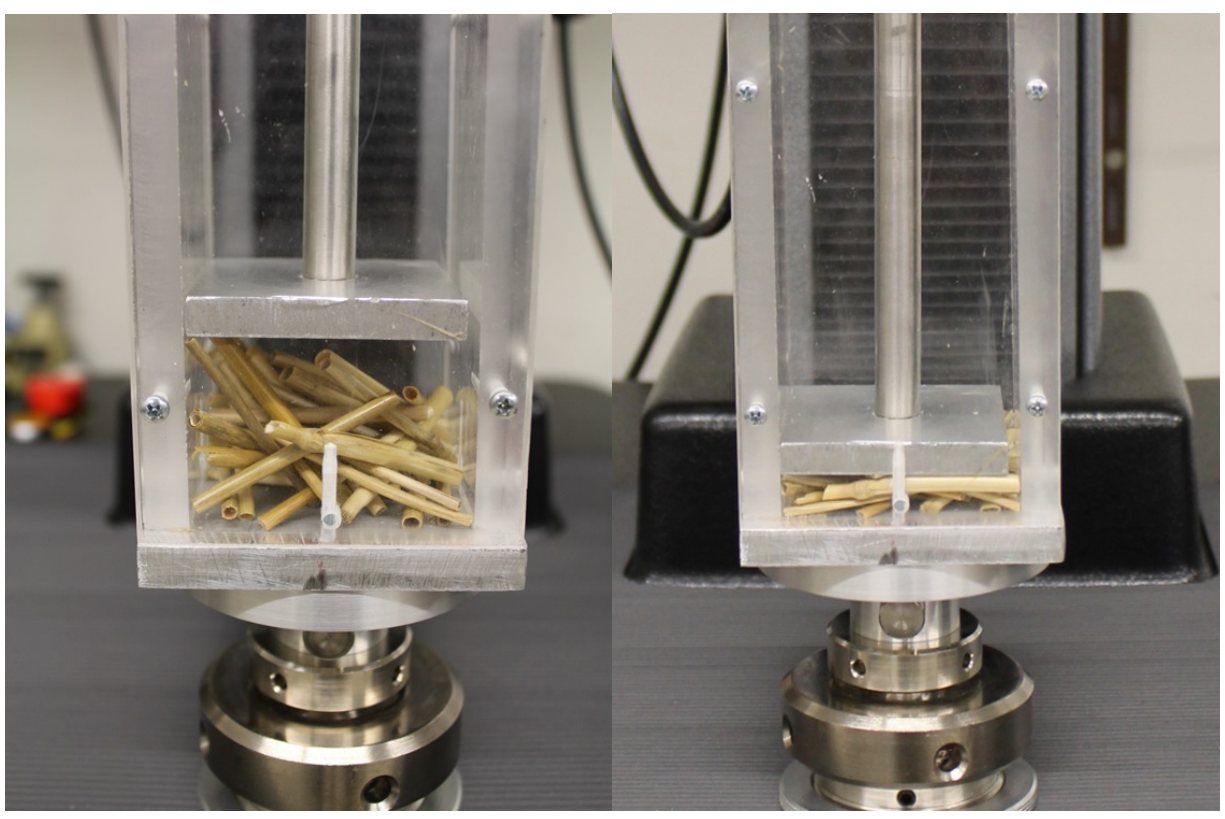

(a)

(b)

Fig. 6. Bulk measurements

(a) stem orientations after initialisation, (b) bulk compression 
where $\sigma$ is the stress, $\epsilon$ is the engineering strain and $m$ and $n$ are empirical crop parameters. The model was fitted separately to the loading and unloading part of the simulated and measured compression profiles.

The validation consists of determining whether the compression model can describe both the simulated and measured compression profiles. When the model parameters for the simulations and the measurements are not significantly different, this indicates that the bulk deformation behaviour of crop stems can be estimated based solely on the stem properties.

\section{Results and discussion}

\subsection{Stem measurements}

Stem measurements were used to create virtual stems with realistic geometries and realistic contact behaviour. Typical force-deformation measurements for stem-plate and stem-stem compression are displayed in Fig. 7. The deformation behaviour is in both cases highly non-linear and plastic.

Thirty wheat stems were measured for both types of compression. Table 2 gives a summary of the stem diameters used in each set-up. These measured stem diameters were also given to the virtual crop stems.

Table 2. Stem diameters (for both compression types thirty wheat stems were measured)

\begin{tabular}{lll}
\hline Compression type & Average stem diameter & Standard deviation on the stem diameters \\
\hline stem-stem & $4.05 \mathrm{~mm}$ & $0.60 \mathrm{~mm}$ \\
stem-plate & $3.98 \mathrm{~mm}$ & $0.59 \mathrm{~mm}$ \\
\hline
\end{tabular}

An example of a typical measured stepwise deformation profile of a wheat 


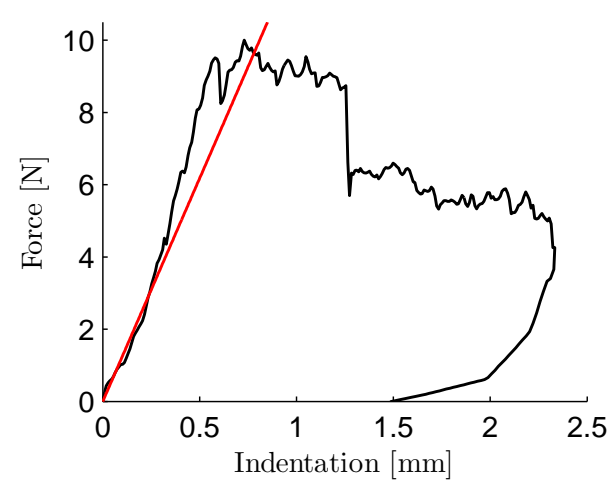

(a)

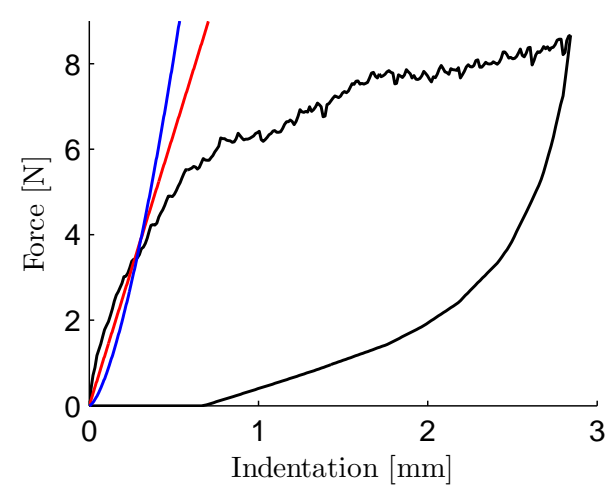

(b)

Fig. 7. Measured compression profiles of wheat stems. The hysteresis is a result of the plastic deformation.

(a) stem-plate interaction, (b) stem-stem interaction.

black: measured data, red: linear elastic model, blue: Hertz model

The interaction of a cylinder with a surface (a) results in a line contact. In this situation, the Hertz model predicts a linear contact force. 


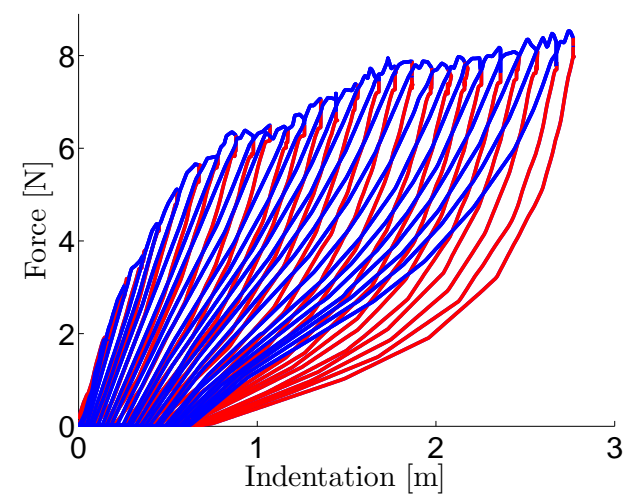

Fig. 8. Stepwise stem-stem deformation (experimental results)

For clarity loading is displayed in blue and unloading is displayed in red

319

stem is displayed in Fig. 8. This stepwise stem-stem measurement shows how the deformation behaviour changes due to plastic deformation. It also shows the reaction to the retraction of the plunger and gives information about hysteresis.

\subsection{Graphical representation of the look up tables}

The stepwise deformation data were used to create look up tables. A graphical representation of the Look up tables (both increasing and decreasing indentation) built from the data in Fig. 8 is shown in Fig. 9. Each horizontal line represents one cycle in the stepwise deformation. Because the MHD is always larger than the indentation/overlap, there are no data in the lower right corner. For all measurements (both stem-plate and stem-stem) look up tables were built. 


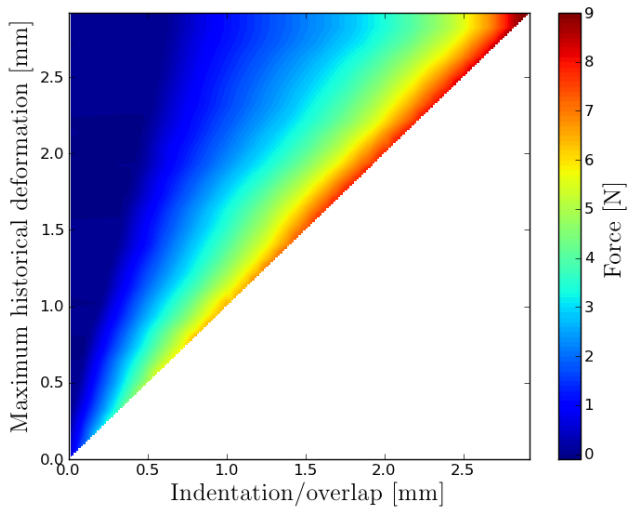

(a)

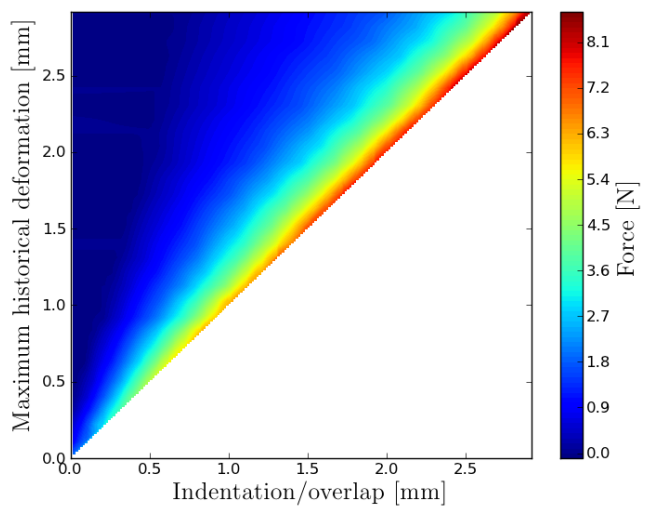

(b)

Fig. 9. Look up tables for the stepwise stem-stem interaction of Fig. 8. A grid size of $0.01 \mathrm{~mm}$ was used. The diameter of the stem was $3.6 \mathrm{~mm}$. The maximum deformation/overlap in these LUT's $(3 \mathrm{~mm})$ thus corresponds to a strain of 0.833 .

(a) Downward movement of the plunger (increasing deformation), (b) Upward movement of the plunger (decreasing deformation) 


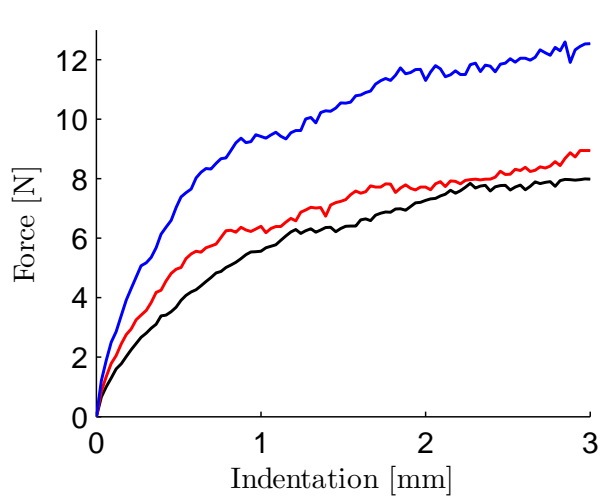

(a)

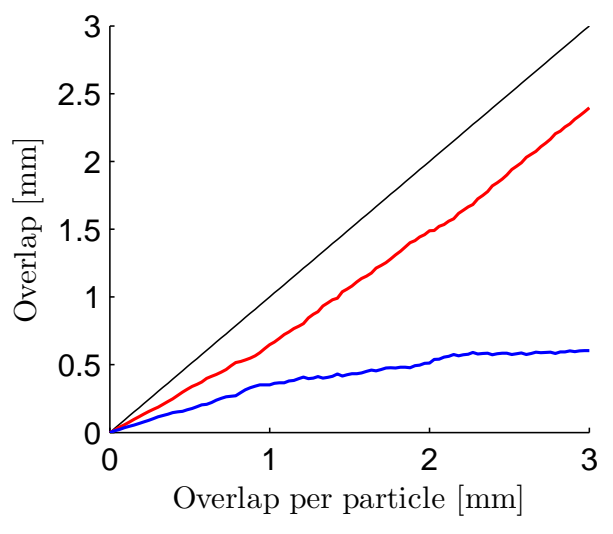

(b)

Fig. 10. Distribution of the overlap of the two interacting stems and resultant forces (Eq.

(a) black: resulting contact force, blue: force-deformation profile of the first stem, red: force-deformation profile of the second stem (b) black: total overlap, blue: overlap of the first stem, red: overlap of the second stem,

\subsection{Contact forces}

In Fig. 10(a) the force-deformation curves of two stems with equal diameter (red and blue) are displayed. The contact force (the force which both particles exert on each other during interaction) is displayed in black. In accordance with Hertzian theory, the contact force is smaller than the force required to deform each stem separately. The contact force corresponds most with the deformation force of the easiest deforming stem (red). If a particle does not (or very little) deform, the contact force will be equal to the deformation force of the other particle. In Fig. 10(b) the distribution of the overlap is displayed. The stem that deforms most easily (red) has the largest overlap. The sum of both overlaps (red and blue) is equal to the total overlap (black). 


\subsection{Stem simulations}

To validate the contact model stepwise compression measurements were conducted on wheat stems. These measurements were repeated in simulation. In Fig. 11(a) a typical stem-plate measurement (black) and the corresponding simulation (blue) are displayed. To demonstrate that the LUT's can generate forces corresponding to MHD's and indentations that were not measured, a different step size was used in the simulations and in the measurements. A typical stem-stem measurement (black) and the corresponding simulation (blue) are displayed in Fig. 11(b). It is clear that the simulations correspond well with the measurements. This was expected since the stem deformation model is data based. A similar result was obtained for all thirty stem-stem measurements and for all thirty stem-plate measurements.

In an analytical model, the same degree of accuracy would require many parameters to be estimated. A data based model is more accurate and less computationally expensive. However, it is less generic. Stem Properties for new conditions must be measured since they cannot be calculated from the model.

\subsection{Bulk compression}

To validate the contact model, bulk compression measurements were conducted on randomly orientated stems (Fig. 12(a)). These measurements were also simulated with the data based contact model (Fig. 12(b)). For clarity, only one measured stress-strain profile and the best corresponding simulation profile are displayed in Fig. 12(c). For both compression profiles the bulk model (Eq. (8)) is plotted (both for loading and unloading). 


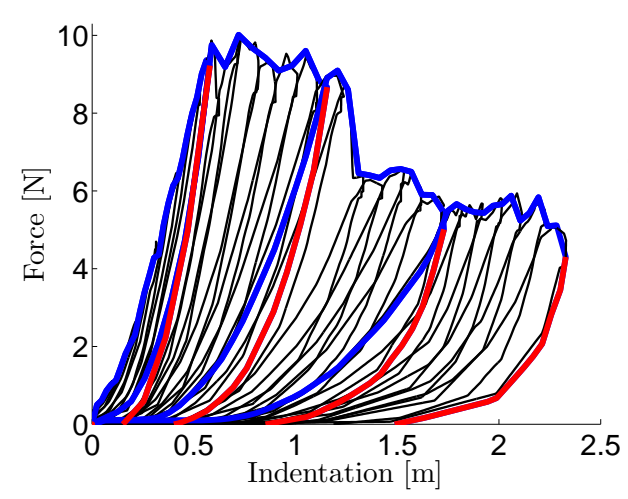

(a)

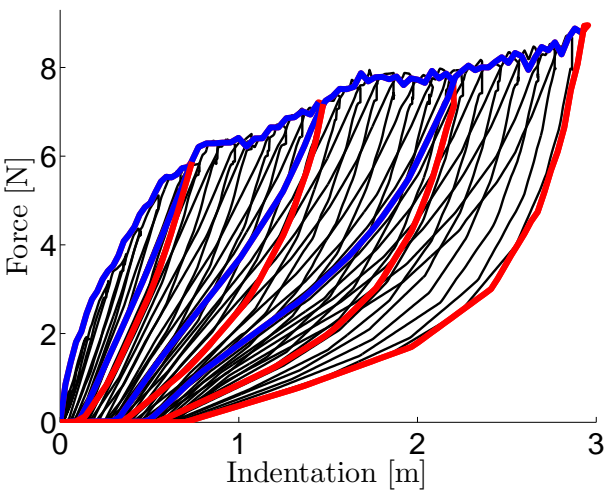

(b)

Fig. 11. Stem compression (black: measurement, blue: simulation (loading), red: simulation (unloading))

(a) stem-plate compression, (b) stem-stem compression

The values of the model parameters, obtained by fitting Eq. (8) to all stress-strain profiles, are summarized in Table 3. From the large standard deviations $(\sigma)$ of the model parameters (both for $m$ and $n$ and both for loading and unloading ), it becomes clear that large variations exists in the measured force-deformation behaviour, even for stems from the same field. This is partly due to different stem characteristics, but also due to different stem orientations after initialization. The same phenomena can be observed in the simulations. The $R^{2}$-values are high both for the measured and the simulated compression profiles indicating that both can be described using the existing compression model.

The values of the fitted parameter $m$ (used to describe the bulk compression) are not significantly different for the measurements and simulations (p-values from the t-tests: 0.194 (loading) and 0.415 (unloading)). The pvalues indicate that the t-test does not reject the null hypothesis (the dif- 


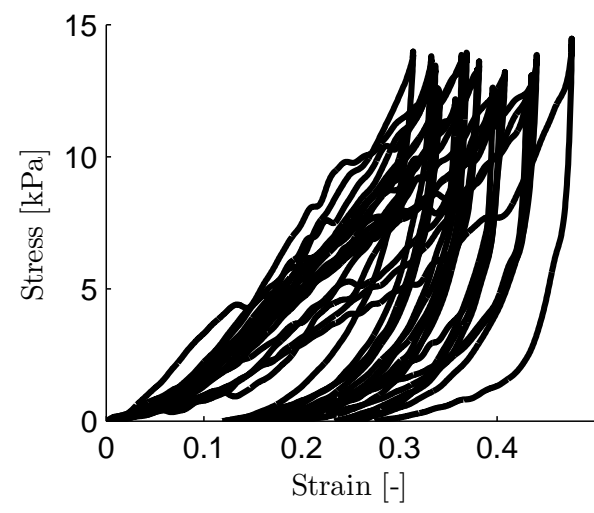

(a)

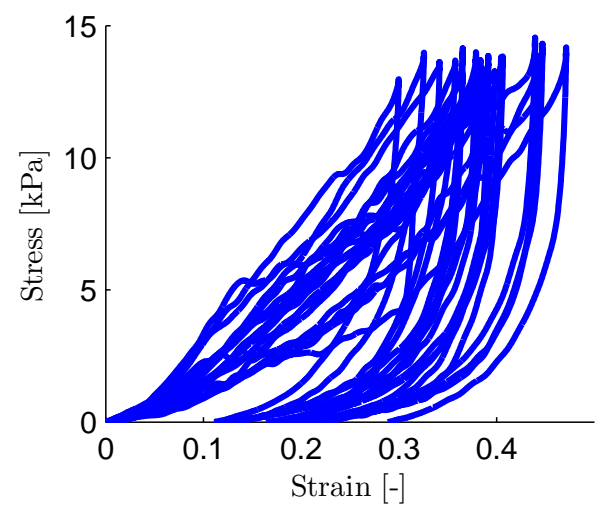

(b)

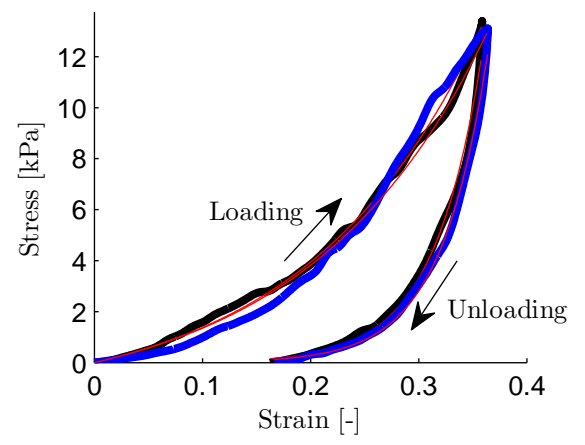

(c)

Fig. 12. Bulk compression (black: measurements, blue: simulations with LUT's) (a) all measured bulk profiles, (b) all simulated bulk profiles,(c) one measured and one simulated profile and the corresponding bulk models (red) 
Table 3. Estimated bulk crop parameters for both the simulations and the measurements $(\mathrm{N}$ : amount of samples, $\mathrm{m}$ and $\mathrm{n}$ : model parameters (Eq. (8)), $\mu$ : mean, $\sigma$ : standard deviation)

\begin{tabular}{lccccccccc}
\hline & $\mathrm{N}$ & \multicolumn{1}{c}{$m$} & & \multicolumn{2}{c}{$R^{2}$} & & \\
& & $\mu$ & $\sigma$ & $\mu$ & $\sigma$ & $\mu$ & $\sigma$ \\
\hline Measurement & & & & & & & \\
\hline Loading & 20 & 4.156 & 2.150 & 5.182 & 2.572 & 0.995 & 0.006 \\
Unloading & 20 & 0.148 & 0.108 & 22.452 & 2.365 & 0.996 & 0.005 \\
\hline Simulation (data based contact model) & & & & & & & \\
\hline Loading & 20 & 3.532 & 2.313 & 4.663 & 1.983 & 0.995 & 0.005 \\
Unloading & 20 & 0.229 & 0.136 & 22.581 & 2.956 & 0.989 & 0.008 \\
\hline
\end{tabular}

ferences between the values of $m_{\text {measurements }}$ and the values of $m_{\text {simulations }}$ have a mean equal to zero) at the $5 \%$ significance level. The same is true for parameter $n$ (p-values for the comparison of measurements and simulations: 0.284 (loading) and 0.804 (unloading)). If the differences are not significant, no distinction can be made between the measurements and the simulations. Therefore, the bulk deformation behaviour of crops can be determined based on the properties of individual stems using databased contact models and DEM simulations.

It should also be noted that, for the simulation of the processing of crops in agricultural machinery not only realistic compression models but also realistic bending and friction models are required. Stems which exhibit different compression behaviour will indeed also bend differently. The biological variability should therefore also be taken into account. In addition, Leblicq et al. (2015b) showed that the bending behaviour of crop stems is highly nonlinear and plastic. A data-based approach could also offer a solution to this 
problem. Also the influence of friction (both between individual stems and between stems and machine components) should be studied in more detail.

\section{Conclusions}

Linear elastic and Hertzian contact models can approximate interaction forces between tubular particles for small deformations. However, as harvesting machines cause larger deformations, realistic simulations can only be obtained if the behaviour for larger deformations is also realistic. Therefore, contact models need to realistically describe interactions over the entire deformation range. To meet this need, in this study data based contact models were developed for crop stems. For this purpose, look up tables were created from stem measurements. These measurements were performed stepwise to assess the influence of plastic deformation. To determine the contact forces between stems, the virtual overlap was divided over both interacting particles such that the individual deformation forces are equally large. The interaction between individual stems and between stems and plates was simulated and validated with measurements on both stem and bulk level. A good correlation was found between both.

\section{Acknowledgements}

The authors wish to thank Dirk Leroy for constructing the test set-up. Also thanks to IWT / KU Leuven IOF for support in the development of Demeter++. 


\section{References}

Favier, J., Abbaspour-Fard, M., Kremmer, M., \& Raji, A. (1999). Shape representation of axi-symmetrical, non-spherical particles in discrete element simulation using multi-element model particles. Engineering Computations, 16, 467-480.

Geng, F., Li, Y., Wang, X., Yuan, Z., Yan, Y., \& Luo, D. (2011). Simulation of dynamic processes on flexible filamentous particles in the transverse section of a rotary dryer and its comparison with video-imaging experiments. Powder Technology, 207, 175-182.

Grof, Z., Kohout, M., \& Štěpánek, F. (2007). Multi-scale simulation of needle-shaped particle breakage under uniaxial compaction. Chemical engineering science, 62, 1418-1429.

Grof, Z., \& Štěpánek, F. (2013). Distribution of breakage events in random packings of rodlike particles. Physical Review E, 88, 1-5.

Guo, Y., Curtis, J., Wassgren, C., Ketterhagen, W., \& Hancock, B. (2013a). Granular shear flows of flexible rod-like particles. POWDERS AND GRAINS 2013: Proceedings of the 7th International Conference on Micromechanics of Granular Media, 1542, 461-494.

Guo, Y., Wassgren, C., Hancock, B., Ketterhagen, W., \& Curtis, J. (2013b). Validation and time step determination of discrete element modeling of flexible fibers. Powder Technology, 249, 386-395.

Guo, Y., Wassgren, C., Ketterhagen, W., Hancock, B., \& Curtis, J. (2012a). 
Some computational considerations associated with discrete element modeling of cylindrical particles. Powder Technology, 228, 193-198.

Guo, Y., Wassgren, C., Ketterhagen, W., Hancock, B., James, B., \& Curtis, J. (2012b). A numerical study of granular shear flows of rod-like particles using the discrete element method. Journal of Fluid Mechanics, 713, 1-26.

Haff, P., \& Werner, B. (1986). Computer simulation of the mechanical sorting of grains. Powder Technology, 48, 239-245.

Janert, P. K. (2013). Feedback Control for Computer Systems. O'Reilly Media Inc. Sebastopol, CA. USA.

Jünemann, D., Kemper, S., \& Frerichs, L. (2013). Simulation of stalks in agricultural processes applications of the discrete element method. Landtechnik, 68, 164-167.

Kajtar, p., \& Loebe, S. (2014). Diskrete element simulation von halmgut. In Land Technik AgEng 2014.

Kattenstroth, R., Harms, H., \& Lang, H. (2011). Systematic alignment of straw to optimise the cutting process in a combines straw chopper. In Proceedings of Land. Technik AgEng 2011.

Leblicq, T., Vanmaercke, S., Ramon, H., \& Saeys, W. (2014). A discrete element model for realistic bendable straw. In Proc. Int. Conf. Agricultural Engineering, AgEng2014, 6.-10.07.2014, Zurich. Eur. Soc. Ag. Eng. (EurAgEng). 
Leblicq, T., Vanmaercke, S., Ramon, H., \& Saeys, W. (2015a). Discrete element modelling of bendable tubes. International Journal of Mechanical Sciences, 94-95, 75-83.

Leblicq, T., Vanmaercke, S., Ramon, H., \& Saeys, W. (2015b). Mechanical analysis of the bending behaviour of plant stems. Biosystems Engineering, 129, 87-99.

Lenaerts, B., Aertsen, T., Tijskens, E., De Ketelaere, B., Ramon, H., De Baerdemaeker, J., \& Saeys, W. (2014). Simulation of grain-straw separation by discrete element modeling with bendable straw particles. Computers and Electronics in Agriculture, 101, 24-33.

Mohsenin, N. N. (1986). Physical properties of plant and animal materials. Gordon and Breach, Science Publishers, Inc.

Nan, W., Wang, Y., Ge, Y., \& Wang, J. (2014). Effect of shape parameters of fiber on the packing structure. Powder Technology, 261, 210-218.

Nguyen, D. H., Kang, N., \& Park, J. (2013). Validation of partially flexible rod model based on discrete element method using beam deflection and vibration. Powder Technology, 237, 147-152.

O'Dogherty, M. (1989). A review of the mechanical behaviour of straw when compressed to high densities. Journal of Agricultural Engineering Research, 44, 241-265.

Rasmussen, K. J. (2003). Full-range stress-strain curves for stainless steel alloys. Journal of constructional steel research, 59, 47-61. 
482 Ross, R. F., \& Klingenberg, D. J. (1997). Dynamic simulation of flexible 483 fibers composed of linked rigid bodies. The Journal of chemical physics, $484 \quad 106,29-49$.

485 Tijskens, E., Ramon, H., \& De Baerdemaeker, J. (2003). Discrete element 486 modelling for process simulation in agriculture. Journal of sound and vi487 bration, 266, 493-514. 